\title{
Controversies about the adjustment of gravitational valves in respect to a new device Christoph Miethke*1, Christian Sprung ${ }^{2}$ and Michael Kiefer ${ }^{3}$
}

\author{
Address: ${ }^{1} \mathrm{GmbH} \&$ Co.KG, Ulanenweg 2, 14469 Potsdam, Germany, ${ }^{2}$ Neurosurgical Department University of Berlin, Charité, Germany and \\ ${ }^{3}$ Neurosurgical Department University of Homburg, Germany \\ Email: Christoph Miethke* - christoph.miethke@miethke.com \\ * Corresponding author
}

from 50th Annual Meeting of the Society for Research into Hydrocephalus and Spina Bifida

Cambridge, UK. 30 August - 2 September 2006

Published: 21 December 2006

Cerebrospinal Fluid Research 2006, 3(Suppl I):S2 doi:10.1 I86/I743-8454-3-SI-S2

(c) 2006 Miethke et al; licensee BioMed Central Ltd.

\section{Background}

The essential drawback of available adjustable devices for the treatment of hydrocephalus is the fact that the adjustment is valid for both positions of the patient. If the opening pressure is increased to decrease the risk of over drainage in the upright position of the patient the same adjustment leads to a very high opening pressure while the patient is lying and consequently to an under drainage in the horizontal position. On the other hand the lowering of the opening pressure automatically increases the risk of subdural effusion as soon as the patient is in upright position.

\section{Materials and methods}

A new device has been developed to introduce the possibility of a valve adjustment, which is effective only in the upright position of the patient. Hereby it is possible to ensure a very low opening pressure of the device in the horizontal position and an adjustment of the high-pressure chamber for the upright position depending on the needs of the individual patient. The adjustment of the valve works similar to the proGAV, which introduces a mechanical brake to avoid unintended readjustments of the device. To establish a posture depending function of the valve the gravity of a titanium disc acting on a sapphire ball defines the opening pressure of the upright position. The weight of this titanium disc is counterbalanced by an adjustable spring-force. At the lowest adjustment the whole weight of the titanium disc acts against the sapphire ball, at the highest adjustment the whole weight is coun- terbalanced and the gravity of the titanium disc has no impact on the opening pressure of the valve.

\section{Results}

The new device fulfils the physical requirements for a shunt to differentiate between the horizontal and vertical position of the patient. The adjustment of the valve pressure independent of the posture of the patient is important especially for patients with untypical intraabdominal pressures, for female patients during pregnancy or patients with unclear clinical outcome.

\section{Conclusion}

The new device offers new possibilities especially for patients with NPH and children. 